\title{
Spin polarized photoelectrons with unpolarized light in normal emission from $\mathrm{Pt}(110)$
}

\author{
N. Irmer *, F. Frentzen, B. Schmiedeskamp, U. Heinzmann \\ Universität Bielefeld, Fakultät für Physik, D-33615 Bielefeld, Germany
}

(Received 20 August 1993)

\begin{abstract}
The normally emitted photoelectrons of the reconstructed $(1 \times 2)$ surface of $\mathrm{Pt}(110)$ caused by off-normally incoming, unpolarized light are spin polarized. Measurements have been performed with the light of a gas discharge tube and spin polarization of up to $20 \%$ has been found for the spin polarization perpendicular to the reaction plane, defined by the momenta of incoming photon and outgoing electron beam. The polarization depends significantly on a rotation of the crystal about the surface normal. The experimental data are in good agreement with theoretical calculations of Feder's group [1] based upon the theory describing the photoemission in the "one-step model".
\end{abstract}

\section{Introduction}

Spin-resolved photoemission spectroscopy has proved to be a successful method to receive information about the electronic structure of nonmagnetic crystals [2-5]. Experiments which investigated the normally emitted photoelectrons from centrosymmetric crystals have been mainly carried out with circularly polarized light, since linearly polarized or unpolarized light should yield no spin polarization in the generally applied "three-step model" of the photoemission process [6] which decomposes the photoemission process into bulk excitation, transport to and transmission through the surface.

\footnotetext{
* Corresponding author.
}

The experimental results of this paper belong to a series of works in which the photoemission process is studied for cases where spin polarization is only expected in the "one-step model" of photoemission while it is forbidden for reasons of symmetry in the "three-step model" [7-13]. That such cases indeed exist was predicted in Ref. [7] and for the first time experimentally verified in Ref. [8] where normally incident linearly polarized light has ejected spin-polarized photoelectrons normal to the three-fold surface of Pt(111). Experiments and theory with an Au overlayer on Pt(111) [9] have revealed that the sign and the size of the spin polarization depend sensitively on the geometrical surface structure. The same experiment with two-, four- or six-fold surfaces is expected to yield no spin polarization because of symmetry reasons. Spin polarization occurs for by far more general conditions if the light incidence 
is off-normal. According to symmetry considerations $[10,11]$ spin-polarized electrons can then even be expected with unpolarized radiation and for practically each surface symmetry $[10,11]$. Analytical calculations in the "one-step model" for (111) surfaces [10] and (100) surfaces [11] show that the spin polarization vector $\boldsymbol{P}$ is perpendicular to the reaction plane which is defined by photon and photoelectron momenta. $|\boldsymbol{P}|$ scales as $|\boldsymbol{P}| \propto \sin 2 \theta \cdot \operatorname{Im}\left(m_{\|} m_{z}^{*}\right)$ and is independent of the azimuthal angle $\Phi[10,11]\left(m_{\|}\right.$and $m_{z}$ are the transition matrix elements for excitation with the components of the $\boldsymbol{E}$-vector parallel and perpendicular to the crystal surface and $\theta$ is the angle between the incoming light and the emitted photoelectrons). The spin polarization is due to spin-orbit induced hybridization of initial states with different symmetry types of their spatial parts. A similar mechanism is well known in the photoionization of free atoms and molecules where spin-polarized photoelectrons can also be obtained with unpolarized radiation $[14,15] . P$ is then also perpendicular to the reaction plane and $|\boldsymbol{P}| \propto \sin 2 \theta \cdot \operatorname{Im}\left(D_{1} D_{2}^{*}\right) \quad\left(D_{1}\right.$ and $D_{2}$ denote complex transition matrix elements for transitions into different final states).

Experiments have been carried out with different photon energies for the three-fold surfaces of $\mathrm{Pt}(111)$ and $\mathrm{Au}(111)$ [12] and for the four-fold surface of $\mathrm{Pt}(100)$ [13]. The spectra obtained reveal spin polarization of up to $30 \%$ in the photoemission peaks for direct transitions which indeed does not change if the crystal is rotated about the surface normal. For two-fold surfaces a corresponding line of symmetry arguments as in Ref. [11] allows a change of the spin polarization vector $\boldsymbol{P}$ with $\Phi$. Therefore, measurements with such surfaces would be a further good test of the theory of the spin polarization effect. In addition, a systematic study of the different cases in which spin polarization occurs only in the "one-step model" but not in the "three-step model" of photoemission is of great interest since experimental results and theoretical calculations have shown that these effects are sensitive to the geometrical structure of the surface [9], the surface potential barrier [10], the relaxation of the surface [11] and the reconstruction of the surface [1]
Furthermore, measurements yield possibly even information needed for a more realistic treatment of the surface optical response [1].

\section{Experiment}

The experimental set-up is shown in Fig. 1. All experiments have been carried out with the clean $(1 \times 2)$ reconstructed surface of a $\mathrm{Pt}(110)$ single crystal. The surface was prepared by sputtering, heating in oxygen and flashing. It was characterized by low-energy electron diffraction (LEED) and Auger-electron spectroscopy (AES). A gas discharge tube produced the unpolarized radiation which hit the crystal surface at an angle of $62^{\circ}$ with respect to the surface normal. For our experiments the discharge tube worked with the noble gas argon which yielded a photon energy of $11.8 \mathrm{eV}$ (Ar I radiation). The normally emitted photoelectrons were energy analyzed by an electrostatic analyzer. After passing through a deflector and a lens system they were accelerated towards the gold foil of the Mott detector. Due to Mott scattering, two components of the spin polarization vector could be determined in the Mott detector. $P_{y}$ is perpendicular to the reaction plane and is therefore the interesting component for the measurements. The other one, $P_{z}$, is parallel to the surface normal of the crystal and has been equal to zero for all our measurements.

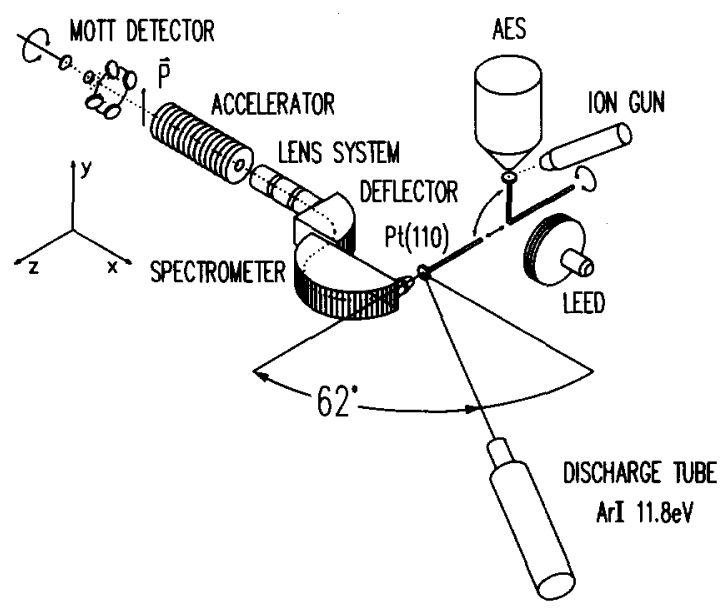

Fig. 1. Experimental set-up. 


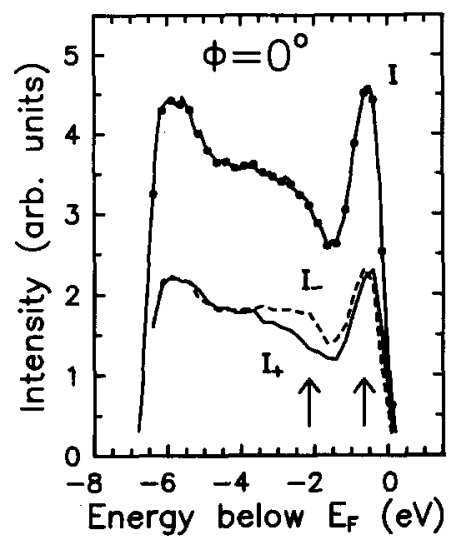

Fig. 2. Spin-resolved photoemission spectrum obtained with unpolarized $11.8 \mathrm{eV}$ radiation for normal emission from a $(1 \times 2)$ surface of $\operatorname{Pt}(110)$. The azimuthal angle $\Phi$ was $0^{\circ} . I$ denotes the total (non-spin resolved) intensity; $I_{+}$and $I_{-}$are the partial intensities with spin up and down, respectively. The arrows at -2.15 and $-0.65 \mathrm{eV}$ indicate the energetic positions for which the dependence of $P_{y}$ on the azimuthal angle $\Phi$ was determined.

In order to investigate the azimuthal dependence of the spin polarization, we have rotated the crystal about the surface normal. The clean surface of the $\mathrm{Pt}(110)$ single crystal reconstructs in a stable $(1 \times 2)$ reconstruction, the so-called "missing row model" which means that every other close-packed row in the first monoatomic layer is missing. This reconstruction retains the two-fold symmetry of the point group $\mathrm{C}_{2 \mathrm{v}}$. The azimuthal angle $\Phi$ has been defined such that for $\Phi=0^{\circ}$ the missing rows are perpendicular to the reaction plane while for $\Phi=90^{\circ}$ they are parallel to the reaction plane.

\section{Experimental results}

Figs. 2 and 3 show the experimental results obtained for the $(2 \times 1)$ surface of $\operatorname{Pt}(110)$ and a photon energy of $11.8 \mathrm{eV}$. The two spin-resolved photoemission spectra were taken at different azimuthal angles, $\Phi=0^{\circ}$ and $\Phi=90^{\circ}$. Dots together with the solid line represent the total intensity $I$ which is split up into the two curves of the partial intensities $I_{+}$(solid curve) and $I_{-}$ (dashed curve). $I_{+}$and $I_{-}$indicate the number

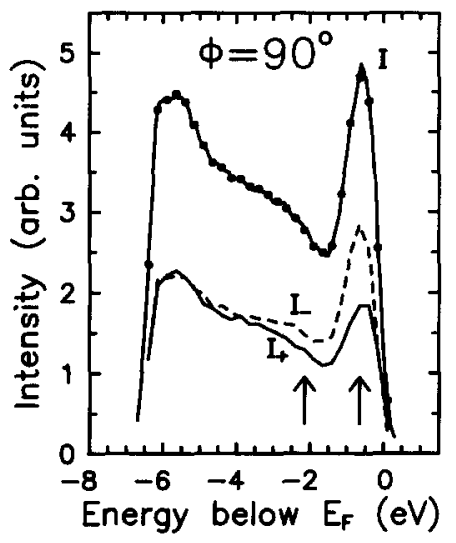

Fig. 3. Spin-resolved photoemission spectrum as in Fig. 2. The azimuthal angle $\Phi$ was $90^{\circ}$.

of electrons with spin parallel and antiparallel to the $y$-direction and can be determined by means of the equation $I_{ \pm}=\frac{1}{2} I \cdot\left(1 \pm P_{y}\right)$. Concerning the spin polarization the spectra for different $\Phi$ differ strongly from each other. While for both azimuthal angles negative spin polarization is found between 2 and $4 \mathrm{eV}$ below $E_{\mathrm{F}}$, the spectrum for $\Phi=90^{\circ}$ reveals a strong negative spin polarization of up to $-20 \%$ in the peak area which is absent in the spectrum for $\Phi=0^{\circ}$. This means that there is a dependence of the polarization on a rotation of the crystal about the surface normal. To investigate this azimuthal dependence

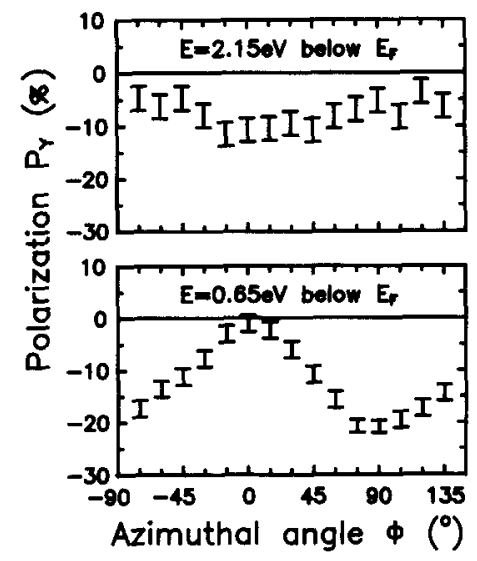

Fig. 4. Dependence of the spin polarization $P_{y}$ on the rotation $\phi$ of the $\operatorname{Pt}(110)$ crystal about the surface normal for two different energies $\left(\Phi=0^{\circ}\right.$ : missing rows perpendicular to the reaction plane). 
more deeply we have changed the azimuthal angle $\Phi$ and have measured the spin polarization for the two energies $2.15 \mathrm{eV}$ and $0.65 \mathrm{eV}$ below $E_{\mathrm{F}}$ (Fig. 4) (the energies are indicated by arrows in the spin-resolved spectra of Figs. 2 and 3). For $2.15 \mathrm{eV}$ below $E_{\mathrm{F}}$ only a slight variation of the spin polarization with the azimuthal angle $\Phi$ can be noticed, whereas for $0.65 \mathrm{eV}$ below $E_{\mathrm{F}}$ the variation is very strong and changes from $0 \%$ to $-20 \%$. This curve has a sinusoidal behaviour with a periodicity of $180^{\circ}$ and therefore reflects distinctly the two-fold symmetry of the crystal.

\section{Discussion}

The data prove that spin polarization with off-normally incoming unpolarized light occurs even for two-fold systems like $\mathrm{Pt}(110)$. This is not surprising since the basic requirement for the occurrence of the effect is fulfilled, namely the existence of hybridized bands (experiments with circularly polarized light have shown that the initial bands of platinum along the $\Sigma$ direction are highly hybridized $[16,17])$. Contrary to the previous experimental results for three- and four-fold surfaces, an azimuthal dependence of the spin polarization has experimentally been found.

Theoretical calculations in the "one-step model" for the $(1 \times 2)$ reconstruction of $\mathrm{Pt}(110)$ have already been made by Scheunemann et al. [1] and they are in good agreement with our experimental data. The calculated spin polarization is, as in our experiments, very small for $\Phi=0^{\circ}$ and large for $\Phi=90^{\circ}$. Furthermore, calculated spectra for the $(1 \times 1)$ reconstruction of $\mathrm{Pt}(110)$ lead to different results [1] and that shows that the spin polarization values are not only sensitive to the azimuthal angle but also to the surface reconstruction of the crystal.

In conclusion, spin-resolved photoemission experiments in normal emission from the two-fold system of $\mathrm{Pt}(110)$ have been carried out with off-normally incoming unpolarized light. A strong azimuthal dependence of the spin polarization which reflects the two-fold symmetry of the crystal has been found. The experimental data are in good agreement with theoretical calculations [1]. The measurements support the theory of the spin polarization effect which occurs under very general conditions and which is only allowed in the "one-step model" of photoemission.

\section{Acknowledgements}

We would like to thank R. Feder and J. Henk for making their calculations available to us and for many valuable discussions. The work was financially supported by the Deutsche Forschungsgemeinschaft (SFB 216).

\section{References}

[1] T. Scheunemann, J. Henk and R. Feder, Bessy Jahresbericht 1992 (1993) 219.

[2] U. Heinzmann, Phys. Scr. T 17 (1987) 77.

[3] F. Meier and D. Pescia, in: Optical Orientation, Eds. F. Meier and B.P. Zakharchenya (North-Holland, Amsterdam, 1984) p. 295.

[4] J. Kirschner, Polarized Electrons at Surfaces (Springer, Berlin, 1985).

[5] M. Wöhlecke and G. Borstel, in: Optical Orientation, Eds. F. Meier and B.P. Zakharchenya (North-Holland, Amsterdam, 1984) p. 423.

[6] R. Feder, in: Polarized Electrons in Surface Physics, Ed. R. Feder (World Scientific, Singapore, 1985) p. 125.

[7] E. Tamura, W. Piepke and R. Feder, Phys. Rev. Lett. 59 (1987) 934.

[8] B. Schmiedeskamp, B. Vogt and U. Heinzmann, Phys. Rev. Lett. 60 (1988) 651.

[9] P. Stoppmanns, B. Heidemann, N. Irmer, N. Müller, B. Vogt, B. Schmiedeskamp, U. Heinzmann, E. Tamura and R. Feder, Phys. Rev. Lett. 66 (1991) 2645.

[10] E. Tamura and R. Feder, Solid State Commun. 79 (1991) 989.

[11] E. Tamura and R. Feder, Europhys. Lett. 16 (1991) 695.

[12] B. Schmiedeskamp, N. Irmer, R. David and U. Heinzmann, Appl. Phys. A 53 (1991) 418.

[13] N. Irmer, R. David, B. Schmiedeskamp and U. Heinzmann, Phys. Rev. B 45 (1992) 3849.

[14] U. Heinzmann, G. Schönhense and J. Kessler, Phys. Rev. Lett. 42 (1979) 1603.

[15] U. Heinzmann, in: Fundamental Processes in Atomic Collision Physics, Eds. H. Kleinpoppen, J.S. Briggs and H.O. Lutz (Plenum, New York, 1985) p. 269.

[16] J. Garbe, D. Venus, S. Suga, C. Schneider and J. Kirschner, Surf. Sci. 178 (1986) 342.

[17] D. Venus, J. Garbe, S. Suga, C. Schneider and J. Kirschner, Phys. Rev. B 34 (1986) 8435. 